\title{
Nephrology at the Time of Nephron's Inception in 1964: An Eye-Opener on Progress in the Field
}

\author{
Leon G. Fine \\ Editor-in-Chief Nephron Journals, Cedars Sinai Medical Center, Los Angeles, Calif., USA
}

\section{Introduction}

As individuals, we tend to look back at what our recent memories tell us. A long-term view is more difficult to gauge. The 50th anniversary of Nephron is an eye-opener when one looks into where our field lay a half-century ago. This was the time when the word 'nephrology' began to be used to describe the broad range of interest in the function of the kidney and body fluids and the diseases thereof. In some respects, progress has been spectacular; in others it has been remarkably lacking. This sobering realization emerges from the brief 'look-back' at where we were when the journal Nephron was born in 1964.

The creation of the journal Nephron half a century ago was not one of those events that arose when a few people with similar interests said 'let's start a new journal to encourage broader interest in our field of endeavor'. Rather, its roots lay at the very foundation of nephrology, as a discipline seeking an international presence. When I assumed the editorship of Nephron a decade ago, I summarized briefly the birth of the journal, calling it 'the original journal of nephrology' [1]. (There were one or two minor start-up journals in this field which never took root, so Nephron indeed led the way.) These origins were the outcome of the establishment, in 1960, of the International
Society of Nephrology (ISN), by Jean Hamburger in Paris. The new society decided to create an international journal of nephrology to be called Nephrologia. The publisher was to be Karger Editions of Basel, the current publisher of Nephron journals.

After a few years of haggling, a name change to Nephron was agreed, and Gabriel Richet of Paris and George Schreiner in Washington, D.C., were to be its founding Editors-in-Chief. At the time of writing of this editorial, Professor Richet is alive and well and living in Paris.

The ISN had held its first congress in 1960 in Evian, France, where the word 'nephrology' was used for the first time in a formal context. The proceedings of the Congress were published in a relatively large tome by S. Karger (Basel and New York) in 1961 [2].

The purpose of this editorial is to try to understand what was on the minds of the founders of the discipline of nephrology around the time when the first issues of Nephron started to roll off the press in 1964. Articles from the earliest issues of the journal were undoubtedly reflective of the key interests of the times, but to make the analysis more inclusive, I have referred to the proceedings of the first ISN Congress in 1960, when a broad range of topics was in evidence (individual contributions are not referenced separately). What follows is a personal view of

\section{KARGER}

E-Mail karger@karger.com

www.karger.com/nec
(C) 2014 S. Karger AG, Basel

1660-2110/14/1261-000I\$39.50/0
Leon $\mathrm{G}$. Fine

Editor-in-Chief Nephron Journals

Cedars Sinai Medical Center

Los Angeles, CA 90048 (USA)

E-Mail Leon.fine@cshs.org 
the field based upon presentations made at this seminal gathering of the world's leading authorities. Obviously, the topics covered reflect the interests of academically minded clinicians and of renal scientists rather than those of practicing physicians, but then, this is what scientific publications deal with.

Broadly speaking, the field of nephrological investigation was focused upon the functioning of the normal kidney, the pathophysiology of kidney disease, and therapeutic developments.

\section{The Functioning of the Normal Kidney and Its Homeostatic Role}

It is intriguing to note that the most substantial 'brainpower' in nephrology in the 1960s lay in the area of renal physiology [2]. The mysteries of the renal concentrating mechanism were beginning to be solved by Wirz, Hargitay, Kuhn and Ryffel in Europe. The countercurrent exchange in blood vessels and the countercurrent multiplier in the loops of Henle allowed the hypertonicity of the renal medulla to be explained. Berliner in the USA formulated his ideas on the mechanisms of formation of concentrated and dilute urine based upon the emergence of observations on single nephron function, using micropuncture techniques. The intellectually challenging nature of this problem attracted some of the best minds in the field of renal physiology. Model systems were proposed where there were very few observational data to support or refute them.

Pari passu with this focus were the data arising from micropuncture studies of the proximal tubule, which set the stage for understanding the nature of the fluid delivered into the loops of Henle, and hence a prediction of how the loop should function. This was the beginning of the era when hypotheses and model systems could be tested by direct measurements made on the nephron in vivo. Arguably, renal physiology had begun to move from the 'black box' of clearance studies of whole organ function into those of single nephron function. Transport systems were beginning to be defined, but nowhere was there the mention of the molecular events involved.

This was also the time when radiolabeled ions and molecules were deployed, and kidney tissue analysis was used to define active and passive transport systems and the driving forces behind them. Luminaries of this era were Berliner, Gottschalk, Lassiter, Levinsky, Ullrich, Morel and others in the USA and Europe [2].
Interest in acid-base metabolism was intense and was led by clinical investigators who were able to conduct extensive studies in human subjects, which would make many contemporary nephrologists envious. Schwartz, Relman and colleagues were analyzing the mechanisms of acidosis and alkalosis and were able to infer the critical roles of ammonia and bicarbonate excretion in these syndromes [2]. Experimental models, combined with carefully performed balance studies, allowed for an understanding of clinical presentations in patients with normal and diseased kidneys, which remains entirely relevant and applicable today.

\section{The Diseased Kidney}

At the forefront of interest in the eyes of physicians trying to understand the pathophysiology of acute renal failure were animal models which mimicked its clinical presentation. At that time, the basis for the reduction in glomerular filtration rate and renal blood flow were the primary foci of interest [2]. The 'shocked' kidney in humans was the model against which animal models had to be compared.

Fifty years ago, electron microscopy was just beginning to emerge as a valuable tool for understanding what was happening at the glomerular capillary level in various forms of primary glomerular disease. The concept of glomerular sieving of macromolecules was beginning to be supported by the beautiful electron micrographs generated by Marilyn Farquar [2]. Not only was the fine structure of the glomerular capillary in evidence, with its unique cell types (including the controversial question of whether or not a 'mesangial' cell exists), but it could be seen precisely where large molecules were restricted from passing through the glomerular capillary wall. It was at this time that effacement of the foot processes of the glomerular epithelial cell was first observed in experimental animals receiving albumin infusions.

At that time it was becoming apparent that the chronically diseased kidney functioned in a very different way from the acutely injured kidney. Following upon the earlier observations of Platt in the UK, Bricker and colleagues in the USA were highlighting the prowess of the surviving nephrons of chronically diseased kidneys to adapt and to allow patients with reasonably well-maintained body fluid compositions to survive until renal function had fallen to very low levels [3]. The notion at the time was that factors extrinsic to the kidney were the controllers of such adaptations, but the means to separate these from intrin-
II

Nephron Clin Pract 2014;126:I-IV DOI: $10.1159 / 000358433$
Fine 
sic nephron adaptations, including renal hypertrophy, were not available. The causes of primary glomerular diseases and understanding the progressive nature of kidney diseases received virtually no attention 50 years ago.

\section{Therapeutic Innovations}

\section{Pharmacology}

The means available for altering the function of the normal and the diseased kidney were extremely limited 50 years ago. Indeed the principal focus was on diuretics, where interest lay more in how and where such drugs worked in the nephron than how they should be used in clinical situations [2]. Orloff showed the site of diuretic action of cardiac glycosides to be on the tubular basolateral sodium-potassium exchanger. Laragh was able to conclude that thiazide diuretics probably have their site of action in the distal tubule. The notion that tight blood pressure control in hypertension or blood glucose level control in diabetes could be attainable therapeutic goals was nowhere in evidence.

\section{Dialysis}

In the 1960s, dialysis as a therapeutic option had been persuasively shown by Merrill in the USA, in the context of acute renal failure. He had the experience of over a decade of using the artificial kidney in such situations, but his belief at the time was that, if profound oliguria was present for more than 10 days, it was not reversible and that dialysis should be discontinued. Others, such as Alwall in Sweden, disagreed based upon experience with a small number of patients who had survived for months on dialysis.

One of the most illuminating insights emerging around the time of Nephron's origin relates to the emergence of chronic dialysis as the means to sustain life in patients with chronic renal disease. In a very short communication to the INS Congress in 1960, which is humbling to contemplate today, Scribner, Hegstrom and Buri from Seattle provided a 'progress report' to the gathered delegates on the treatment of chronic uremia by means of hemodialysis [2]. They cannulated the radial artery and forearm veins, connected by a special coupling bypass device, in 4 patients who were dialyzed for a total of just under $29 \mathrm{pa}-$ tient-months (each for around $24 \mathrm{~h}$ once a week). Continuous flow dialysis was used. The early experience of clotting, infection and kinking of the catheters was noted. The problem of bubbles forming in the venous line after prewarming of the blood had not been solved. A hyperosmotic bath was used to effect fluid removal of 2-4 liters during the 24-hour dialysis. The case report alluded to the problems of hypertension, weight loss and hyperkalemia, but serum creatinine concentrations, which had been at around $14-16 \mathrm{mg} / \mathrm{dl}$, fell to $6-8 \mathrm{mg} / \mathrm{dl}$ after dialysis.

It is as if Everest had been scaled for the first time, and the challenge had been laid down for others to follow. Remarkably, the focus on renal physiology by academic leaders in nephrology at the time probably diverted interest from this groundbreaking discovery by regarding it as a technological advance of little interest to those trying to solve the deeper conceptual problems.

\section{Renal Transplantation}

A second and parallel set of events was emerging at the time, i.e. the beginnings of renal transplantation [2]. Two centers were gaining experience under prominent leaders in the field: Jean Hamburger in Paris and John Merrill in Boston. By 1960, John Merrill had performed 17 transplantations with only 1 survivor (for 5.5 months), because chronic dialysis was not an option at the time. He was struggling to understand how to suppress the lymphoid reaction to foreign antigens. In a well-known transplantation between twins performed by Joseph Murray (who later received a Nobel Prize for his contributions to tissue transplantation), the recipient survived without immune suppression. Merrill was exploring how best to manage the response to foreign antigens from nonidentical donors. He assumed that rejection was immunological in nature and argued that if functioning lymphoid tissue could be suppressed, a state of 'tolerance' would ensue. Utilizing total body irradiation, followed by pooled bone marrow administration, this approach was used until the advent of prednisone therapy. Merrill was left with having achieved 'partial tolerance' and presciently believed that this would eventually also become applicable to the treatment of glomerulonephritis.

Simultaneous with these events across the Atlantic, Jean Hamburger at the Hôpital Necker in Paris was contending with the same issues. $\mathrm{He}$, too, found that the transplantation within the same family improved survival dramatically and, based upon 'inbreeding' experiments, thought that there must be 'at least 15 transplantation genes' in mice. He went on to try to develop a blood test as a predictive test for rejection.

Hamburger was trying to weaken a patient's ability to reject a graft, using chemotherapeutic and immunotherapeutic approaches. Irradiation of a fraternal twin recipient prior to transplantation allowed for survival of the kidney for 7 months. The protocol was tried on nontwin patients, leading Hamburger to prophesy: 'The ancients 
invented the chimera, a fabulous animal composed of a lion's head, a goat's body and a dragon's tail. Will chimeric men exist in the future? It is too early to answer. Nevertheless, in Paris and Boston there are few young men or women alive today who would have died without kidney transplantation. Only that should encourage us to pursue this endeavor.'

\section{Conclusions}

From the foregoing we can conclude that, since the 1960 s, in some areas we have made breathtaking advances and in some virtually none at all. We now do understand more or less how the kidney works as an organ. After 50 years of trying, only now are we seeing a glimmer of understanding of how primary glomerular diseases are caused at a molecular level, but such insights have yet to find their ways into clinical practice. The vast majority of diseases which lead to end-stage renal disease remain poorly understood and seem unlikely to be solved in the coming decade despite the existence of the most sophisticated genomic and other biological information.

In this context, it is illuminating to take a look at a single area of interest, chronic renal failure, and to consider how it evolved during the mid-1960s. (It is possible to gauge this from a publication of the American Society of Nephrology which appeared in 1992 in celebration of 25 years of the society's existence [4].)

In that period, investigators were focused upon functional adaptations of the diseased kidney, as alluded to above, and upon the uremic syndrome and uremic toxicity. There was no interest in mechanisms of progression of kidney disease; this was completely absent from publications in the 1960s and only surfaced in the early 1980s.
With respect to understanding uremic toxins, what they are and how they function, the field has been static. Despite occasional favored toxin molecules popping up from time to time, uremic toxicity is still poorly understood. In the area of pathophysiology and mechanisms of adaptation of the diseased kidney, the advances have been more tangible, in that expertise and understanding of the basic mechanisms were revealed by disease models. It became possible to understand where in the nephron adaptations were localized and, more importantly, how they are regulated [3]. Certain areas of great interest at that period seem to have quietly disappeared, one example being the role of a natriuretic hormone as a regulator of sodium handling by the diseased kidney.

While nephrologists today may be justifiably proud of the vastly improved survival of patients on dialysis and of those with transplanted kidneys, it is sobering to realize that these 'half-way technologies' only exist because we have failed to prevent or halt the progress of chronic renal diseases at an early stage. Each life-prolonging technology comes with its attendant undesired side effects and its enormous costs. Fifty years ago no one would have dreamed of the vast numbers of patients being kept alive on dialysis or by transplantation across the world. Of concern, however, is the fact that we have made virtually no progress in nipping in the bud the primary renal diseases at their earliest stages, thereby preventing them from ever making such sustaining therapies necessary. Only now are candidate molecular targets emerging, which may serve as diagnostic and prognostic markers and, most importantly, as drug targets. When all is said and done, until we understand the cause of each type of kidney disease at a molecular level, progress in preventing such diseases will be very slow.

The next 50 years should be interesting ones for Nephron!

\section{References}

Fine LG: The birth of Nephron, the original journal of nephrology. Nephron Physical 2003:93:1-2.

2 Richet G (ed): Proceedings of the First International Congress of Nephrology. Basel, Karger, 1961.

3 Fine, L, Nord EP, Danovich GM, Kurtz I, Bacallao R: Pathophysiology and nephron adaptation in chronic renal failure; in Schrier RW, Gottschalk GW (eds): Diseases of the Kidney. Boston, Little Brown \& Co, 1988, pp 2985-3018.

\footnotetext{
4 Fine LG, Grantham JJ, Kopple JD: Chronic renal failure; in Tannen RL, Fine LG, Gottschalk CW, Schreiner GE (eds): A Quarter Century of Nephrology, Commemorating American Society of Nephrology's 25th Anniversary. Washington, American Society of Nephrology, 1992, pp 167-175.
} 